\title{
TEM Study of Oxygen Precipitation in Si Wafers with Backside Layers
}

\author{
R. Yu, ${ }^{*}$ X.F. Zhang, ${ }^{*}$ Q. Wang, ${ }^{* *}$ M. Daggubati, ${ }^{* *}$ and H. Paravi**
}

* Materials Sciences Division, Lawrence Berkeley National Laboratory, Berkeley, CA 94720

** Fairchild Semiconductor Corporation, 3333 West 9000 South, West Jordan, UT 84088

Silicon single crystals are known as the most perfect material that mankind has ever created. Even in such a perfect material, the concentration of oxygen as an impurity is generally higher than those of doping impurities, and they critically affect the electrical and mechanical properties of Si wafers. Therefore, oxygen in silicon has been being one of the hottest subjects in the field of silicon-based materials science and technology [1,2]. In the present work, we study the oxygen precipitation in $\mathrm{Si}$ wafers as a method to remove oxygen from the Si matrix. The precipitation behavior of oxygen in two kinds of backside treatments are compared, i.e. the deposition of polycrystalline Si back layer and the damaged back surface by sand blasting.

The wafers were made of Czochralski silicon crystals and were heavily arsenic doped, with resistivity at $3 \mathrm{~m} \Omega \cdot \mathrm{cm}$ and interstitial oxygen concentration of $7 \times 10^{17} / \mathrm{cm}^{3}$. After the deposition of the polycrystalline Si layer of about $1.3 \mu \mathrm{m}$ thick, or damaged with sand blasting, the wafers were annealed at $1200^{\circ} \mathrm{C}$ for 45 minutes and $900^{\circ} \mathrm{C}$ for $15 \mathrm{hrs}$ under nitrogen ambient. Cross-sectional TEM samples were examined using JEM3010 and CM200-FEG microscopes, operating at $300 \mathrm{keV}$ and $200 \mathrm{keV}$, respectively. Images were recorded digitally with $\mathrm{CCD}$ cameras.

FIG. 1 (a) and (b) show the morphology and the selected area diffraction pattern for the wafer with backside poly-Si layer. It is found that the oxide precipitates formed only at the poly-Si/substrate interface. The diameter of the precipitates is about $10-20 \mathrm{~nm}$. The poly-Si layer is heavily twinned. No precipitates can be found in the Si substrate and the poly-Si layer. The high resolution (FIG. 1 ((c)) indicates that the precipitates are amorphous silicon oxide columns, which lie in (001) plane, with the longitudinal axis inclined with respect to the electron beam direction [1-10], resulting in elongated shape at the interface.

For the $\mathrm{Si}$ wafer with the backside damaged by sand blasting, oxide precipitates formed as elongated rods extending into the Si substrate, as shown in FIG. 2 (a). The precipitates lie in $\langle 110\rangle$ directions, and are enclosed by the $\{111\}$ planes of Si. The high resolution image of such a precipitate rod is shown in FIG. 2 (b). Due to the heat treatments in nitrogen ambient, Si-O-N precipitate also formed in the substrate, as confirmed by EDS (not shown here) and the extra diffraction spots seen in diffraction pattern (FIG. 2 (c)).

\section{References}

[1] F. Shimura, editor, Oxygen in Silicon, Academic Press, San Diego, 1994.

[2] A. Borghesi et al. J. Appl. Phys. 77 (1995) 4169. 

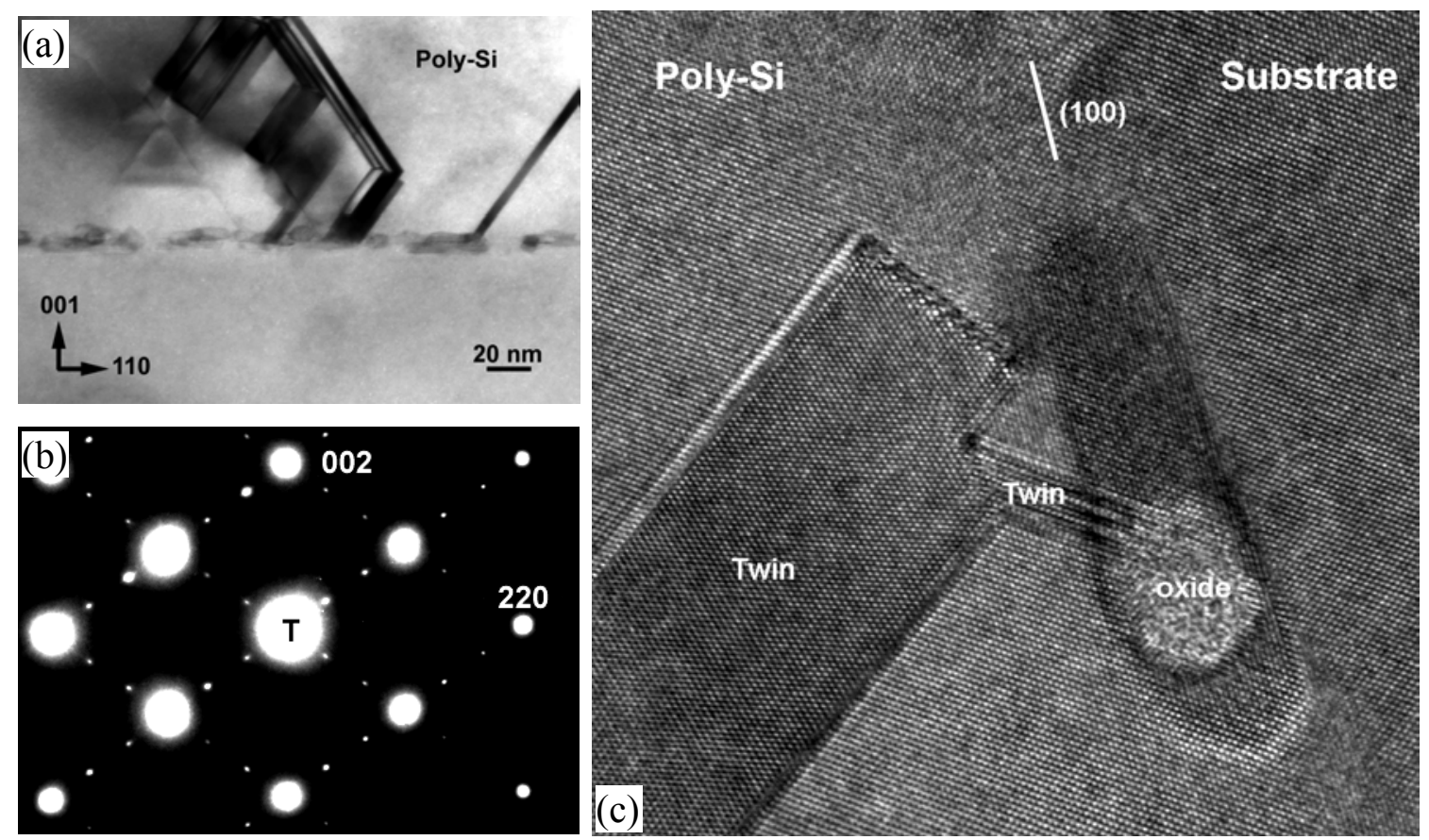

c)

FIG. 1. The wafer with the poly-Si layer: (a) morphology, note the precipitates at the interface; (b) select area diffraction pattern showing the highly twinned poly-Si layer; (c) high resolution image of silicon oxide precipitates at the substrate/poly-Si interface.
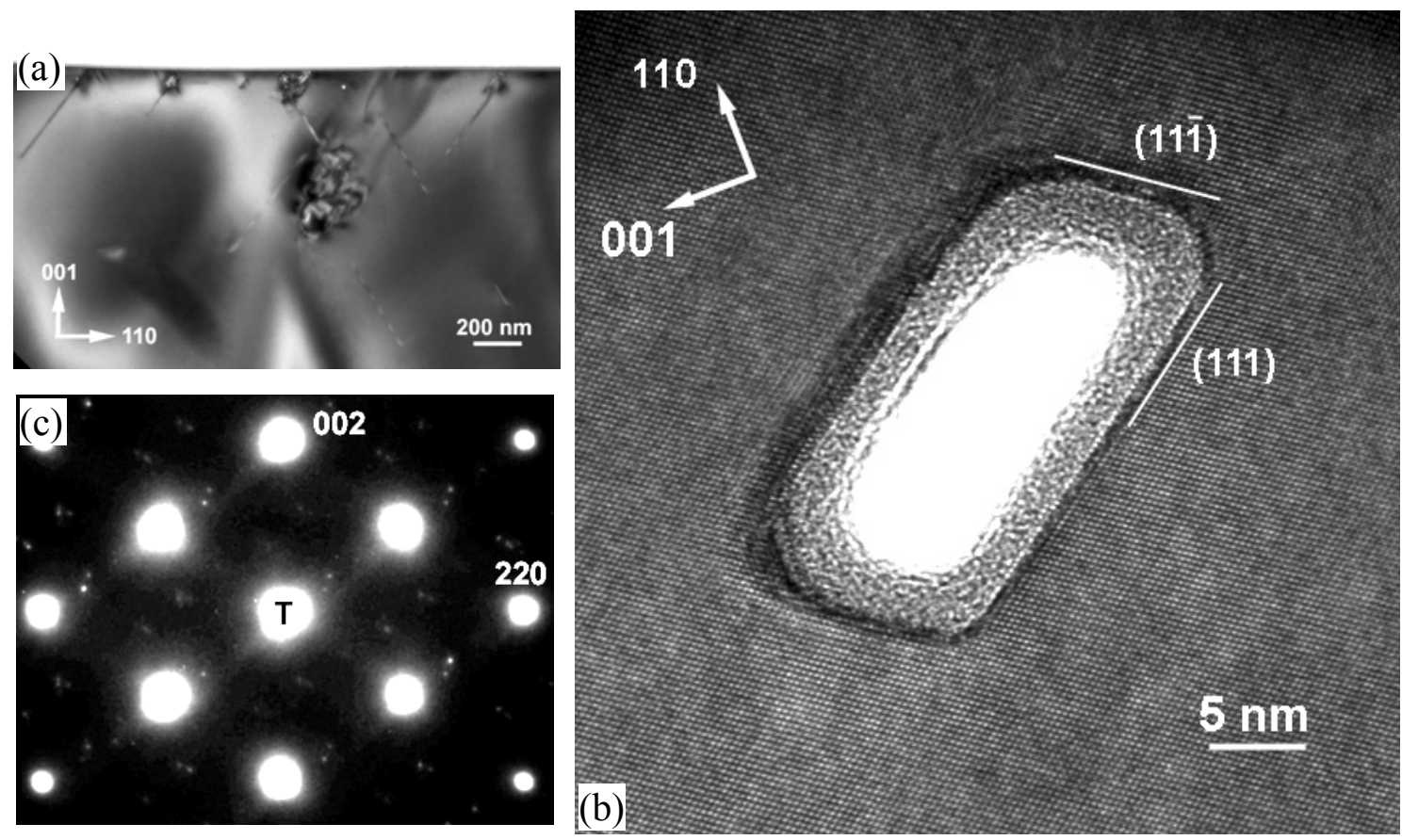

(b)

FIG. 2. The wafer with the backside damaged by sand blasting: (a) morphology; (b) high resolution image of an oxide rod parallel to the electron beam; (c) selected area diffraction pattern showing the formation of Si-O-N precipitates. 